
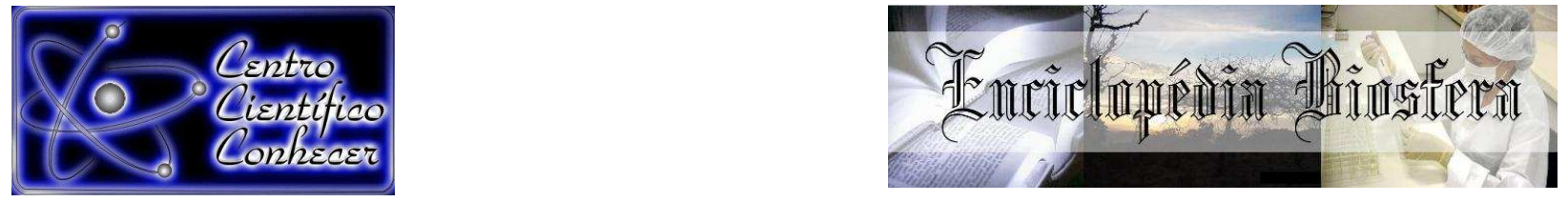

\title{
DISTRIBUIÇÃO E PROBABILIDADE DE PRECIPITAÇÃO PARA NOVA MUTUM, MATO GROSSO, BRASIL
}

Decio Mossini Junior ${ }^{1}$, Henrique da Cruz Ramos ${ }^{2}$, Rivanildo Dallacort ${ }^{3}$, Fabrício Schwanz da Silva ${ }^{4}$.

1. Engenheiro Agrícola, Mestre. Programa de Pós-Graduação em Ambientes e Sistemas de Produção Agrícola, Universidade do Estado de Mato Grosso

(deciomossinijr@hotmail.com).

2. Engenheiro Agrônomo, Mestre. Programa de Pós-Graduação em Ambientes e Sistemas de Produção Agrícola, Universidade do Estado de Mato Grosso.

3. Engenheiro Agrícola, Doutor. Universidade do Estado de Mato Grosso, Departamento de Agronomia.

4. Engenheiro Agrícola, Doutor. Universidade do Estado de Mato Grosso, Departamento de Engenharia de Produção Agroindustrial.

Recebido em: 03/10/2016 - Aprovado em: 21/11/2016 - Publicado em: 05/12/2016 DOI: 10.18677/EnciBio_2016B_039

\section{RESUMO}

O Estado de Mato Grosso tem como principal atividade fonte econômica a agricultura, de modo que o município de Nova Mutum, localizado na Bacia Amazônica é um dos principais produtores de soja do estado. Além disso, no estado predomina a agricultura de sequeiro, onde a cultura tem como única fonte de aporte hídrico a precipitação pluvial, assim o conhecimento sobre esta variável climática é imprescindível para o sucesso das atividades agrícolas. Deste modo, este trabalho busca apresentar a distribuição temporal da chuva e a probabilidade de precipitação para o município de Nova Mutum. Para a elaboração deste estudo foi utilizado um banco de dados de 26 anos de precipitação (1986 a 2011) cedidos pela Agencia Nacional das Águas. Para o cálculo da precipitação provável foram utilizados dois modelos de distribuição, Gama e Log-Normal, em seguida realizado o teste de aderência pra verificar a adequabilidade dos modelos ao banco de dados. A média anual foi de $1.855,9 \mathrm{~mm}$ com um desvio padrão de $399,5 \mathrm{~mm}$, observou-se ainda a presença de duas estações bem definas, uma chuvosa de outubro a março e outra seca entendendo-se de maio a setembro. A distribuição Gama apresentou adequabilidade para cálculo da precipitação provável mensal durante todo o ano, já a Log-Normal apresentou limitações em alguns meses no período de seca.

PALAVRAS-CHAVE: climatologia, precipitação provável, recursos hídricos.

\section{DISTRIBUTION AND PROBABILITY OF PRECIPITATION FOR NOVA MUTUM, MATO GROSSO, BRAZIL}

\section{ABSTRACT}

The agriculture is the main economic activity of the State of Mato Grosso, and the municipality of Nova Mutum, located in the Amazon Basin is a leading producer of soybeans in the state. Being predominant in the state dry farming, where culture has as water sole source the rainfall, thus becomes essential to know about this climate variable for the successful of agriculture activities. Thus this paper aims to present the temporal distribution of rainfall and the probability of precipitation for the municipality under study. For this study was used a database of 26 years of rainfall (1986-2011) donated by the National Water Agency. To calculate the probable ENCICLOPÉDIA BIOSFERA, Centro Científico Conhecer - Goiânia, v.13 n.24; p. 435 
precipitation were used two distribution models, Gamma and Log-Normal, then performed the adhesion test to verify the suitability of the models to the database. The annual average was $1,855.9 \mathrm{~mm}$ with a standard deviation of $399.5 \mathrm{~mm}$, there was still the presence of two seasons, a rainy season from October to March and a dry season from May to September. The Gamma distribution appeared adequate to calculate likely monthly rainfall all year round, but the lognormal presented limitations in some months during the dry season.

KEYWORDS: climatology, hydric resources, probable rainfall.

\section{INTRODUÇÃO}

Localizado na Amazônia Mato-grossense, o município de Nova Mutum, apresenta uma economia voltada principalmente a produção agropecuária (LIMA, 2011), ocupando a quarta colocação no volume de soja produzido no Estado de Mato Grosso (IBGE, 2013), e desta forma, também economicamente dependente das condições climáticas, principalmente ao regime pluviométrico.

Estudar a probabilidade de variáveis climáticas é de grande importância para o planejamento de atividades agropecuárias, construção civil, turismo e transporte (ULIANA et al., 2013). CUNHA et al. (2013) realçam que o conhecimento da precipitação máxima provável para um determinado local, é fundamental para a realização de trabalhos relacionados a conservação do solo, construções de estradas e barragens e preservação dos recursos naturais. Além disto, segundo DALLACORT et al. (2011) e GARCIA et al. (2013), permite um melhor planejamento agrícola, auxiliando no dimensionamento de sistemas de irrigação e na determinação de épocas de semeadura, evitando assim, déficit hídrico e perdas na produtividade, uma vez que, de acordo com MOURA et al. (2015), a precipitação pluvial é o elemento de maior influência nas condições ambientais, exercendo efeito direto sobre a temperatura do ar e do solo, umidade relativa do ar e radiação solar, fatores que em conjunto atuam no crescimento e desenvolvimento das plantas.

Vários modelos matemáticos de probabilidade têm sido utilizados para o estudo da precipitação provável de um determinado local, dentre estes destacam-se os modelos de distribuição Gama e Log-Normal. O modelo de distribuição de probabilidade Gama, proposto por THOM (1958), adequa-se à estimativa provável de precipitação nos mais distintos locais do país, e também fora dele, fato que pode ser comprovado pelos resultados obtidos por PIZZATO et al. (2012), SOUZA et al., (2013), ERSZURKI et al. (2015) e PINTO et al. (2015).

Outro modelo amplamente utilizando o cálculo da probabilidade de precipitação tem sido o modelo de distribuição Log-Normal. JUNQUEIRA JUNIOR et al. (2007) relatam que o modelo é de fácil operacionalidade e tem apresentado bom ajuste as distribuições de precipitação brasileiras. Os autores testaram diferentes modelos de distribuição de probabilidade e concluíram que o modelo Log-Normal foi o que melhor se ajustou a dados históricos de precipitação mensal. Bons ajustes também foram observados por DANFÁ (2011) e SOBRINHO (2014), na distribuição de probabilidade de lâminas de precipitação pluviométrica.

Perante isto, o presente trabalho objetivou analisar a distribuição mensal e anual, e os níveis de probabilidade de ocorrência de chuvas no município de Nova Mutum - MT, assim como, comparar diferentes modelos probabilísticos de distribuição aplicados as séries históricas de precipitação.

\section{MATERIAL E MÉTODOS}

O presente trabalho foi desenvolvido no Laboratório de Meteorologia como parte da avaliação da disciplina de estatística do Programa de Pós-Graduação em ENCICLOPÉDIA BIOSFERA, Centro Científico Conhecer - Goiânia, v.13 n.24; p. 436 2016 
Ambientes e Sistemas de Produção Agrícola, da Universidade do Estado de Mato Grosso. Os dados diários utilizados foram disponibilizados pela Agência Nacional das Águas (ANA), e remetem a um período de 26 anos (1986 a 2011). O posto pluviométrico utilizado está localizado no município de Nova Mutum - MT (Figura 1), na latitude -1329'8,16", longitude - 56043'12" e altitude de $417 \mathrm{~m}$.

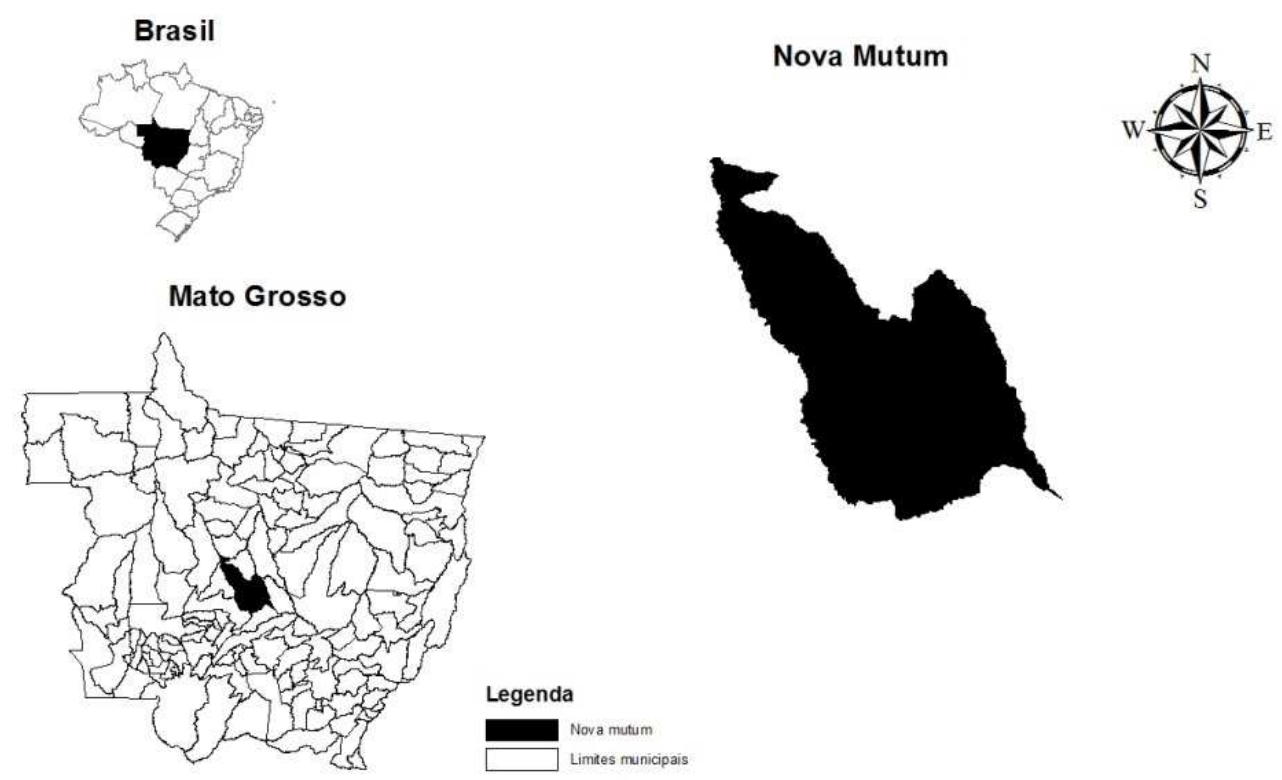

FIGURA 1: Localização espacial do Município de Nova Mutum.

Agrupou-se os dados em intervalos, então foram calculadas as frequências mensais e anual, em seguida determinou-se as médias mensais, anual e o desvio padrão. A quantificação dos dias secos e chuvosos foi realizada conforme o proposto por SANS et al. (2001), considerando como dias secos, os dias com precipitação inferior ou igual a $5 \mathrm{~mm}$ e dias chuvosos, os dias onde a precipitação excedeu este valor.

A precipitação provável foi estimada através das distribuições Gama e LogNormal, nos seguintes níveis probabilísticos: $50,60,75$, e 95\%. Posteriormente os valores estimados foram submetidos ao teste de Kolmogorov - Smirnov ao nível de $5 \%$ de probabilidade, verificando assim quais os modelos adequados para a estimativa da precipitação provável no município de Nova Mutum - MT.

O modelo probabilístico de distribuição Gama incompleto proposto por THOM (1958) (equação 1), aplica-se apenas valores maiores que zero. Quando as séries históricas apresentaram valores iguais à zero utilizou-se distribuição Gama - mista (equação 3) utilizada por RIBEIRO et al. (2001).

$$
f(x)=\frac{1}{\beta^{\alpha} \Gamma(\alpha)} * x^{\alpha-1} e^{-x / \beta}
$$

Onde:

$\alpha=$ parâmetro de forma (adimensional);

$\beta=$ parâmetro de escala $(\mathrm{mm})$;

$x=$ total de precipitação $(\mathrm{mm})$; e

$\Gamma$ = símbolo da função gama, definida conforme equação 2 .

$$
\Gamma=\sqrt{2 \pi \alpha} \alpha^{\alpha} e^{\alpha}\left(1+\frac{1}{12 \alpha}+\frac{1}{288 \alpha^{2}}-\frac{139}{51840 \alpha^{4}}\right)
$$




$$
f(x)=P 0+(1-P 0) G(x)
$$

Sendo:

$$
P 0=\frac{N O}{(N+1)}
$$

Onde:

P0: probabilidade de valores nulos (equação 4);

$G(x)$ : distribuição cumulativa gama;

No: numero de valores nulos; e

$N$ : número de observações.

Os parâmetros alfa e beta foram estimados através do método dos momentos proposto por ASSIS et al. (1996) e utilizado por DALLACORT et al. (2011), estes baseiam-se em equiparar a média $(\bar{X})$ e a variância $\left(S^{2}\right)$ da amostra à média e a variância da população gerando duas equações (equações 5 e 6).

$$
\begin{aligned}
& \alpha=\frac{g^{2}}{\sigma} \\
& \beta=\frac{\sigma}{g}
\end{aligned}
$$

Em que:

$\bar{X}=$ precipitação média do período $(\mathrm{mm})$; e

$\sigma=$ desvio padrão (mm).

A distribuição Log-Normal de 2 parâmetros (Equação 6) foi calculada conforme RIBEIRO et al. (2007) em estudo da precipitação provável para o município de Barbacena-MG.

$$
f(x)=\frac{1}{x * \sigma+\sqrt{2 \pi}} * \exp \left(\frac{\ln (x)-\pi}{\sigma}\right)^{2}
$$

Onde:

$x$ : precipitação do período $(\mathrm{mm})$;

$\sigma:$ desvio padrão $(\mathrm{mm})$; e

$\bar{X}:$ precipitação média $(\mathrm{mm})$.

\section{RESULTADOS E DISCUSSÃO}

O comportamento da precipitação anual do período em questão mostrou grande variação interanual (Figura 2), os valores anuais mínimos e máximos foram respectivamente $1.210,6$ e $2.877,9 \mathrm{~mm}$, uma amplitude de $1.667,3 \mathrm{~mm}$, valores superiores aos observados por PIZZATO et al. (2012) em estudo sobre a precipitação do município de Cáceres - MT onde a amplitude é de apenas 651,2 com os valores variando entre 972,9 e $1.624,1$. Observa-se ainda que $23 \%$ dos anos a precipitação se manteve entre $1.210,6$ e $1.544,1 \mathrm{~mm}$, e em $30,8 \%$, entre $1.544,1$ e $1.877,5 \mathrm{~mm}$. Em outros $30,8 \%$ dos anos, o total precipitado foi de $1.877,5 \mathrm{e}$ $2.210,9 \mathrm{~mm}$ e em apenas $15,4 \%$ dos anos este valor esteve acima de $2.210,9 \mathrm{~mm}$.

A média anual foi de $1.855,9 \mathrm{~mm}$ com um desvio padrão de $399,5 \mathrm{~mm}$. DALLACORT et al. (2011) em estudo realizado para o município de Tangará da 
Serra - MT descreveram uma precipitação anual média de $1.830,8 \mathrm{~mm}$, já MORAES et al. (2005) observaram que cerca de $50 \%$ do estado do Pará possui uma precipitação anual entre 1.800 e $2.300 \mathrm{~mm}$, valores próximos aos observados neste trabalho. Porém, o desvio padrão observado pelos autores foi de 312,8 e $355,8 \mathrm{~mm}$, respectivamente, ambos os valores menores que o calculado para o município de Nova Mutum indicando então uma maior variabilidade das chuvas.

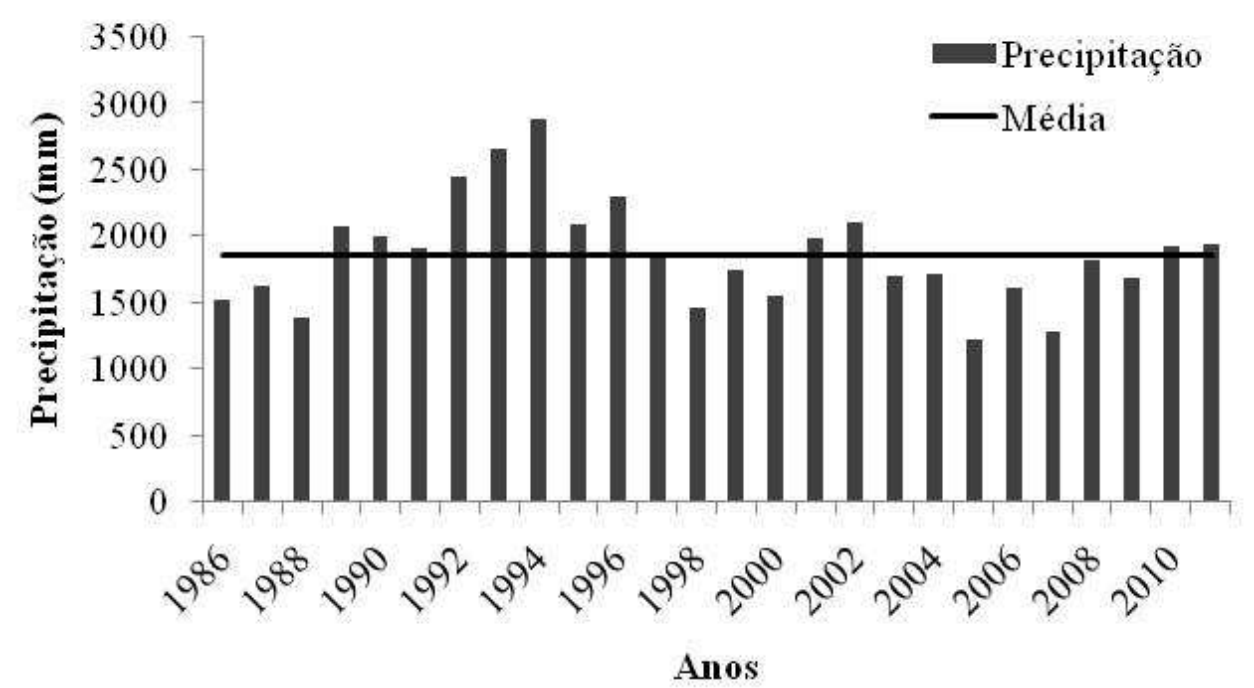

FIGURA 2. Precipitação pluviométrica anual para o município de Nova Mutum MT no período de 1986 a 2011.

As médias de precipitação mensais (Figura 3) seguem um comportamento descrito por MARCUZZO et al. (2011), em que os autores caracterizam a precipitação no Estado de Mato Grosso em um período de chuvas e outro de seca. Os meses de abril e setembro seriam os meses de transição entre o período seco e o chuvoso, e os meses de maio, junho, julho e agosto, compreenderiam o período de seca resultados próximos aos observados por SANTOS et al. (2011) que em estudo para a bacia hidrográfica do Ribeirão João Leite - GO observaram a concentração das chuvas nos meses de outubro a março. Nos meses de dezembro, janeiro e fevereiro (período chuvoso) as médias mensais de precipitação mantiveram-se acima dos $300 \mathrm{~mm}$, já os meses de maio, junho, julho e agosto, apresentam os períodos mais críticos, com precipitação mensal média de $30,1 \mathrm{~mm}$, $20,2 \mathrm{~mm}, 4,5 \mathrm{~mm}$ e $15,5 \mathrm{~mm}$, respectivamente.

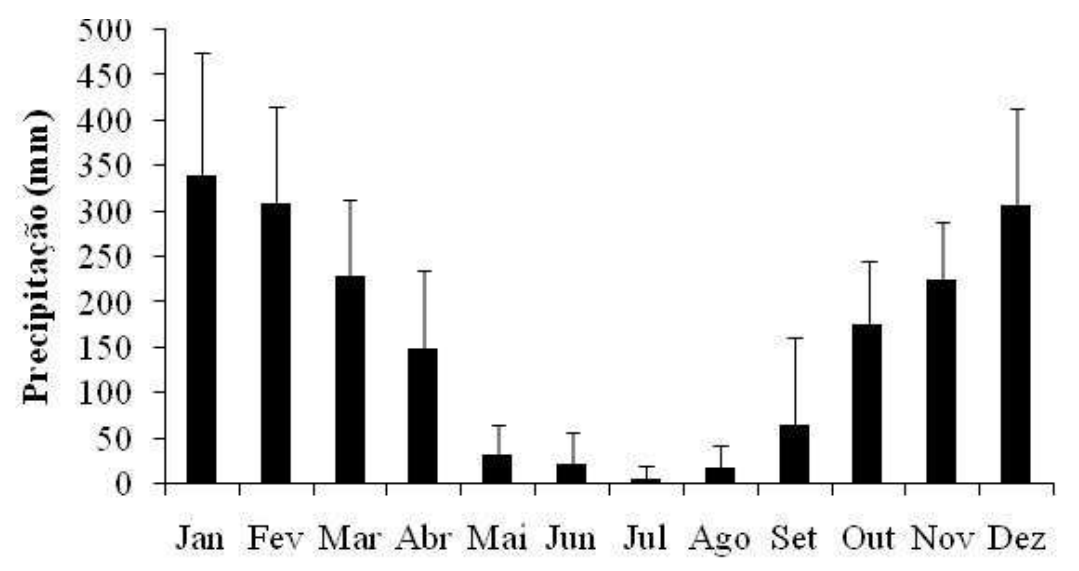

Meses

FIGURA 3. Médias pluviométricas mensais e desvio padrão para o município de Nova Mutum - MT no período de 1986 a 2011. 
Os valores de precipitação média observados nos meses de dezembro, janeiro e fevereiro, para o município de Nova Mutum, apresentaram valores superiores aos encontrados por DALLACORT et al. (2010) para os municípios de Cáceres, Campo Novo do Parecis, Cuiabá, Diamantino e Tangará da Serra, indicando a superioridade da precipitação do município de Nova Mutum aos demais, no período. Nos municípios estudados pelos autores, apenas Campo Novo do Parecis apresentou precipitação maior que $300 \mathrm{~mm}$ no mês de janeiro, porém, inferior a encontrada para o mesmo mês em Nova Mutum.

No período de junho, julho e agosto, a precipitação média mensal, seguiu o mesmo comportamento observado por DALLACORT et al. (2010) para os diferentes municípios estudados (Cáceres, Campo Novo do Parecis, Cuiabá, Diamantino e Tangará da Serra), mantendo níveis de precipitação inferiores a $30 \mathrm{~mm}$.

A média anual de dias chuvosos e dias secos foi 75 e 290 dias respectivamente, de modo que o desvio padrão foi de 9,67 dias para os dias chuvosos, e 9,68 dias para os dias secos. Em estudo sobre a precipitação nas nascentes do Rio Paraguai, MARTINS et al. (2011) observaram no município de Diamantino uma média anual de 88,7 dias chuvosos e 276,3 dias secos. O mês de janeiro, com média de 13,7 dias chuvosos, foi o mês que apresentou maior quantidade de dias chuvosos, seguido pelos meses de fevereiro e dezembro. Já o mês de julho demonstrou o menor número de dias chuvosos, e o maior de dias secos, seguido pelos meses de agosto e junho. O número de dias chuvosos e secos para os meses de junho, julho e agosto foram: 0,8 e 29,2; 0,2 e 30,8; e, 0,7 e 30,3, respectivamente.

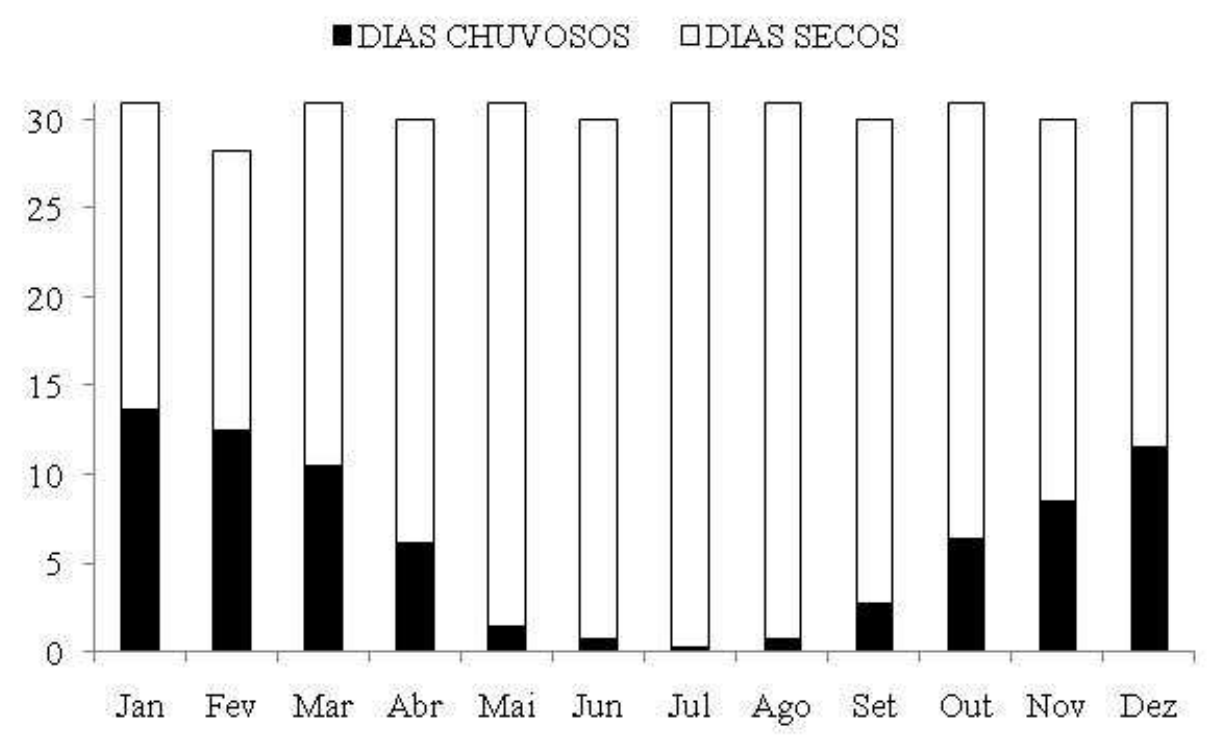

FIGURA 4. Médias mensais de dias secos e chuvosos para o município de Nova Mutum - MT no período de 1986 a 2011.

MARTINS et al. (2011) observaram ainda que para Diamantino os períodos de dezembro, janeiro, e fevereiro, demonstraram os maiores números de dias chuvosos, assim como, junho, julho e agosto, os maiores números de dias secos. Tal comportamento também foi observado para o município de Nova Mutum no presente trabalho, e ainda por MOREIRA et al. (2010) no município de Nova Maringá - MT, município localizado na mesma bacia hidrográfica, que apresentou em média 85 dias chuvosos e 279 dias secos. 
Na Tabela 1 é possível observar que $43,2 \%$ das precipitações ocorridas no ano estão entre 5 e $15 \mathrm{~mm}$, sendo essa a intensidade mais comum em todos os meses do ano, de modo que janeiro é o mês onde estas chuvas mais ocorrem. A segunda intensidade de chuva mais comum foi a de 15 a $25 \mathrm{~mm}$, representando $22,9 \%$ das chuvas ocorridas no ano, os outros $33,9 \%$ estão distribuídos em precipitações mais intensas, acima de 50mm. ARAl et al. (2010) observaram que o potencial erosivo de uma precipitação depende basicamente de dois fatores: intensidade de ocorrência e da energia cinética, deste modo quanto mais intensas as chuvas maior o potencial erosivo.

TABELA 1. Intensidade de precipitação mensal e anual do município de Nova Mutum - MT, no período de 1986 a 2011.

\begin{tabular}{lccccc}
\hline \multirow{2}{*}{ Meses } & \multicolumn{6}{c}{ Intensidade de precipitação ( $\mathbf{m m})$} \\
& $\mathbf{5} \mathbf{~ \mathbf { ~ 1 5 }}$ & $\mathbf{1 5} \mathbf{a} \mathbf{2 5}$ & $\mathbf{2 5}$ a $\mathbf{3 5}$ & $\mathbf{3 5} \mathbf{~} \mathbf{5 0}$ & $\mathbf{>} \mathbf{5 0}$ \\
\hline Janeiro & 5,96 & 3,00 & 1,81 & 1,65 & 1,27 \\
Fevereiro & 5,23 & 2,81 & 1,69 & 1,65 & 1,08 \\
Março & 4,92 & 2,69 & 1,54 & 0,69 & 0,69 \\
Abril & 2,69 & 1,35 & 0,69 & 1,00 & 0,46 \\
Maio & 0,81 & 0,35 & 0,04 & 0,08 & 0,19 \\
Junho & 0,35 & 0,23 & 0,04 & 0,08 & 0,08 \\
Julho & 0,12 & 0,08 & 0,00 & 0,04 & 0,00 \\
Agosto & 0,31 & 0,27 & 0,04 & 0,00 & 0,08 \\
Setembro & 1,54 & 0,54 & 0,08 & 0,19 & 0,42 \\
Outubro & 2,73 & 1,35 & 0,69 & 0,73 & 0,92 \\
Novembro & 3,31 & 1,92 & 1,35 & 0,96 & 0,96 \\
Dezembro & 4,58 & 2,69 & 1,38 & 1,42 & 1,54 \\
Anual & 32,54 & 17,27 & 9,35 & 8,50 & 7,69 \\
\hline
\end{tabular}

Segundo CARVALHO et al. (2013) a maneira como a precipitação é distribuída é de extrema importância para a agricultura, pois a necessidade hídrica diária exigida por uma planta varia de acordo com a espécie, estágio de desenvolvimento e demanda atmosférica. Deste modo, é necessário que haja uma boa distribuição das chuvas durante o desenvolvimento da cultura, visto que, existem períodos onde a necessidade hídrica das plantas é maior, em geral na fase de florescimento e formação dos frutos.

O modelo de distribuição Gama mostrou-se adequado para a estimativa da precipitação para períodos mensais (Tabela 2), o mesmo foi observado por OLIVEIRA et al. (2010), na estimativa da precipitação pluviométrica na cidade de Alegre - ES, por SOCCOL et al. (2010), para o mesmo fim, em Lages - SC e por RIBEIRO \& AVANZI (2010), em Machado - MG. 
TABELA 2. Adequabilidade das distribuições de probabilidade Gama e Log-Normal, para dados de precipitação do município de Nova Mutum - MT, ajustados em período mensal e anual, e avaliados pelo teste de Kolmogorov-Smirnov a 5\% de significância.

\begin{tabular}{lcc}
\hline \multirow{2}{*}{ Meses } & \multicolumn{2}{c}{ Adequabilidade } \\
\cline { 2 - 3 } & Gama & Log-Normal \\
\hline Janeiro & $0,15^{\mathrm{A}}$ & $0,15^{\mathrm{A}}$ \\
Fevereiro & $0,10^{\mathrm{A}}$ & $0,12^{\mathrm{A}}$ \\
Março & $0,18^{\mathrm{A}}$ & $0,22^{\mathrm{A}}$ \\
Abril & $0,11^{\mathrm{A}}$ & $0,15^{\mathrm{A}}$ \\
Maio & $0,14^{\mathrm{A}}$ & $0,12^{\mathrm{A}}$ \\
Junho & $0,23^{\mathrm{A}}$ & $0,48^{\mathrm{NA}}$ \\
Julho & $0,07^{\mathrm{A}}$ & $0,80^{\mathrm{NA}}$ \\
Agosto & $0,25^{\mathrm{A}}$ & $0,59^{\mathrm{NA}}$ \\
Setembro & $0,10^{\mathrm{A}}$ & $0,13^{\mathrm{A}}$ \\
Outubro & $0,14^{\mathrm{A}}$ & $0,17^{\mathrm{A}}$ \\
Novembro & $0,09^{\mathrm{A}}$ & $0,10^{\mathrm{A}}$ \\
Dezembro & $0,10^{\mathrm{A}}$ & $0,08^{\mathrm{A}}$ \\
Anual & $0,11^{\mathrm{A}}$ & $0,10^{\mathrm{A}}$ \\
\hline
\end{tabular}

NA: Não Adequado - Valor calculado maior que o tabelado $(0,26)$ para 0 teste de Kolmogorov-Smirnov $(\alpha=0,05)$; A: Adequado - Valor calculado menor que o tabelado $(0,26)$ para o teste de Kolmogorov-Smirnov $(\alpha=0,05)$;

Já o modelo de distribuição Log-Normal não adequou-se aos períodos de junho, julho e agosto, meses de baixos índices de precipitação na localidade em estudo. Resultados semelhantes foram observados por JUNQUEIRA JUNIOR (2007) e por SILVA et al. (2007). Entretanto, resultados opostos foram observados por SOUZA et al. (2010) que durante a estimativa da precipitação mensal em Pernambuco, obtiveram excelentes resultados utilizando o modelo Log-Normal nos meses de seca.

A distribuição Gama apresentou valores superiores de precipitação quando comparados aos valores obtidos pela distribuição Log-Normal (Tabela 3), com exceção dos períodos de maio e setembro, que intermediam o período seco e chuvoso na região. No período de outubro a março foi observado $90 \%$ de probabilidade de precipitação acima de $100 \mathrm{~mm}$, de acordo com ambos os modelos de probabilidade utilizados. Assim como, a região apresenta $90 \%$ de probabilidade de precipitação anual acima de $1.300 \mathrm{~mm}$. 
TABELA 3. Probabilidade de ocorrência de precipitação a níveis de $90 \%, 75 \%, 60 \%$ e $50 \%$ para o município de Nova Mutum, determinada através de distribuições de probabilidade Gama e Log-Normal.

\begin{tabular}{|c|c|c|c|c|c|c|c|c|}
\hline \multirow[b]{2}{*}{ Meses } & \multicolumn{2}{|c|}{$90 \%$} & \multicolumn{2}{|c|}{$75 \%$} & \multicolumn{2}{|c|}{$60 \%$} & \multicolumn{2}{|c|}{$50 \%$} \\
\hline & Gama & $\begin{array}{c}\text { Log- } \\
\text { Normal }\end{array}$ & Gama & $\begin{array}{c}\text { Log- } \\
\text { Normal }\end{array}$ & Gama & $\begin{array}{l}\text { Log- } \\
\text { Normal }\end{array}$ & Gama & $\begin{array}{l}\text { Log- } \\
\text { Normal }\end{array}$ \\
\hline Janeiro & 178,97 & 172,53 & 238,49 & 227,08 & 286,98 & 274,75 & 319,14 & 308,13 \\
\hline Fevereiro & 181,34 & 181,05 & 230,58 & 226,00 & 269,67 & 263,59 & 295,21 & 289,15 \\
\hline Março & 127,68 & 113,35 & 165,77 & 150,93 & 196,35 & 184,10 & 216,47 & 207,47 \\
\hline Abril & 52,44 & 52,88 & 83,34 & 78,77 & 111,13 & 103,87 & 130,60 & 122,67 \\
\hline Maio & 2,03 & 2,34 & 6,78 & 5,55 & 13,30 & 10,11 & 18,94 & 14,49 \\
\hline Junho & 0,04 & $-^{*}$ & 0,69 & - & 2,89 & - & 5,82 & - \\
\hline Julho & 0,00 & - & 0,00 & - & 0,00 & - & 0,00 & - \\
\hline Agosto & 0,08 & - & 0,82 & - & 2,89 & - & 5,38 & - \\
\hline Setembro & 0,53 & 2,49 & 4,53 & 6,99 & 14,15 & 14,33 & 25,00 & 22,06 \\
\hline Outubro & 93,29 & 85,90 & 123,79 & 114,95 & 148,59 & 140,70 & 165,02 & 158,89 \\
\hline Novembro & 144,78 & 140,03 & 176,34 & 170,67 & 200,79 & 195,78 & 216,55 & 212,63 \\
\hline Dezembro & 180,71 & 197,86 & 229,79 & 237,97 & 268,74 & 270,48 & 294,20 & 292,13 \\
\hline Anual & 1365,9 & 1387,7 & 1573,2 & 1576,5 & 1728,9 & 1722,4 & 1827,4 & 1816,6 \\
\hline
\end{tabular}

*Valores de probabilidade não adequados de acordo com o teste de Kolmogorov-Smirnov ( $\alpha$ $=0,05$ ).

Em linhas gerais a precipitação na cidade de Nova Mutum apresentou o comportamento da precipitação típica do centro-oeste brasileiro, com duas estações bem definidas, seca de maio a setembro e chuvosa de outubro a março. Os períodos de janeiro e dezembro apresentaram maior precipitação e grande variabilidade interanual, demostrando a necessidade de maiores estudos sobre os fenômenos meteorológicos que atuam nessa localidade. O modelo de distribuição Gama, um dos mais utilizados atualmente, apresentou uma adequabilidade para o cálculo da precipitação provável durante todo o ano, já o modelo Log-normal apresentou limitação em seu uso nos meses de seca onde a precipitação se aproxima a zero.

\section{CONCLUSÕES}

O município de Nova Mutum - MT apresentou média de precipitação anual de $1.855,9 \mathrm{~mm}$ com um desvio padrão de $399,5 \mathrm{~mm}$.

A região apresenta duas estações bem definidas, sendo de outubro a abril, chuvosa e de maio a setembro, seca.

A região apresenta $90 \%$ de probabilidade de precipitação anual acima de $1.300 \mathrm{~mm}$, sendo que nos meses de dezembro, janeiro e fevereiro, há $90 \%$ de probabilidade de ocorrência de pluviosidade acima de 170mm.

O método de distribuição Gama, apresentou melhor adequabilidade na determinação da probabilidade de precipitação.

\section{REFERÊNCIAS}

ARAI, F. K.; Gonçalves, G. G. G.; Pereira, S. B.; Comunello, E.; Vitorino, A. C. T.; Daniel, O. Espacialização da precipitação e erosividade na Bacia Hidrográfica do Rio Dourados - MS. Engenharia Agrícola, v.30, n.5, p. 922-931, 2010. Disponível por 
meio de <http://www.scielo.br/pdf/eagri/v30n5/v30n5a14.pdf >. Acessado em 02 de outubro de 2016. DOI: http://dx.doi.org/10.1590/S0100-69162010000500014.

ASSIS, F. N.; ARRUDA, H. V.; PEREIRA, A. R. Aplicações de estatística à
climatologia: teoria e prática. Pelotas: Universidade Federal de Pelotas, 1996. CARVALHO. A. L. SOUZA, J. L.; LYRA, G. B.; PORFIRIO, A. C. S.; FERREIRA JUNIOR R. A. Ocorrência de períodos secos na região de Rio Largo, Alagoas. Revista Brasileira de Meteorologia, Rio de Janeiro, v. 28, n. 2, p. 173-180, 2013. Acesso por meio de <http://www.scielo.br/pdf/rbmet/v28n2/v28n2a06.pdf>. Acessado em 02 de outubro de 2016. DOI: http://dx.doi.org/10.1590/S010277862013000200006 .

CUNHA, M. C.; THOMAZ, E. L.; VESTENA, L R. Medidas de controle de erosão em estradas rurais na bacia do Rio das Pedras, Guarapuava - PR. Revista Soc \& Nat, Uberlândia, v. 25, n. 1, p. 107-118, 2013. Disponível por meio de < http://www.seer.ufu.br/index.php/sociedadenatureza/article/view/14879/pdf>.

Acessado em 02 de outubro de 2016. DOI: http://dx.doi.org/10.1590/S198245132013000100009 .

DALLACORT, R.; MARTINS, J. A., INOUE, M. H.; FREITAS, P. S. L; COLETTI, A. J. Distribuição das chuvas no município de Tangará da Serra, médio norte do estado de Mato Grosso, Brasil. Acta Scientiarum, Maringá, v. 33, n. 2, p. 193-200, 2011. Disponível por meio de <http://www.scielo.br/pdf/asagr/v33n2/01.pdf $>$. Acessado em 02 de outubro de 2016. DOI: http://dx.doi.org/10.4025/actasciagron.v33i2.5838.

DALLACORT, R.; MARTINS, J. A.; INOUE, M. H.; FREITAS, P. S. L; KRAUSE, W. Aptidão agroclimática do pinhão manso na região de Tangará da Serra, MT. Revista Ciência Agronômica, Fortaleza, v. 41, n. 3, p. 373-379, 2010. Acesso por meio de <http://www.ccarevista.ufc.br/seer/index.php/ccarevista/article/view/915/454>. Acessado em 02 de outubro de 2016.

DANFÁ, S.; SILVA, A. M.; MELLO, C. R.; COELHO, G.; VIOLA, M. R.; ÁVILA, L. F. Distribuição espacial de valores prováveis de precipitação pluvial para períodos quinzenais, em Guiné-Bissau. Revista Brasileira de Engenharia Agrícola e Ambiental, Campina Grande, v. 15, n. 1, p. 67-74, 2011. Acesso por meio de <http://www.scielo.br/pdf/rbeaa/v15n1/a10v15n01.pdf>. Acesso em 02 de outubro de 2016. DOI: http://dx.doi.org/10.1590/S1415-43662011000100010.

GARCIA, R. G.; DALLACORT, R.; KRAUSE, W.; SERIGATTO, E. M.; FARIA JUNIOR, C. A. Calendário agrícola para o cutivo de milho em Sinop-MT. Revista Pesquisa Agropecuária Tropical, Goiânia, v. 43, n. 2, p. 218-222, 2013. Acesso por meio

<http://crawl.prod.proquest.com.s3.amazonaws.com/fpcache/6c9d040c6eb111769f0 6038997c98108. pdf?AWSAccessKeyld=AKIAJF7V7KNV2KKY2NUQ\&Expires=1475 471385\&Signature $=4 \mathrm{C} \% 2 \mathrm{~B} 0 \mathrm{MQzVRoIV} \% 2 \mathrm{~F} 6 f s c q 8 \mathrm{ap} \% 2 \mathrm{~F} 70 N W I \% 3 \mathrm{D}>$. Acessado em 02 de outubro de 2016. DOI: 10.1590/S1983-40632013000200014.

IBGE - Instituto Brasileiro de Geografia e Estatística. Culturas temporárias e permanentes. Produção agrícola Municipal, Rio de Janeiro, v. 40, p. 102, 2013. Disponível por meio

de 
$<\mathrm{ftp}: / / \mathrm{ftp}$.ibge.gov.br/Producao_Agricola/Producao_Agricola_Municipal_[anual]/2013/ pam2013.pdf>. Acessado em 02 de outubro de 2016.

JERSZURKI, D.; SOUZA, J. L. M.; EVANGELISTA, A. W. P. Distribuiçãode probabilidade e movimento temporal da precipitação na região de Telemaco Borba, Paraná. Revista Brasileira de Ciências Agrárias, Recife, v. 10, n. 1, p. 110-116, $2015 . \quad$ Acesso por meio de <https://www.researchgate.net/profile/Jorge_Souza8/publication/275275190_Distribui cao_de_probabilidade_e_movimento_temporal_da_precipitacao_na_regiao_de_Tele maco_Borba_Parana/links/5536c8c20cf268fd0018762a.pdf>. Acessado em 02 de outubro de 2016. DOI: 10.5039/agraria.v10i1a5159.

JUNQUEIRA JUNIOR, J. A.; GOMES, N. M.; MELLO, C. R.; SILVA, A. M. Precipitação provável para a região de Madre de Deus Alto Rio Grande: modelos de probabilidade e valores característicos. Revista Ciências e Agrotecnologia, Lavras, v. 31, n. 3, p. 842-850, 2007. Acesso por meio de <http://www.scielo.br/pdf/cagro/v31n3/a34v31n3.pdf>. Acessado em 02 de outubro de 2016. DOI: http://dx.doi.org/10.1590/S1413-70542007000300034.

LIMA, D. M. D. F. Geopolítica do desenvolvimento capitalista na Pré-amazônia Matogrossense: poder e capital na área de influência da BR-163. Revista Sociedade e Território, Natal, v. 23, n. 1, p. 59-74, 2011. Acesso por meio de < http://www.cchla.ufrn.br/revset/index.php/revset/article/view/1/4>. Acessado em 02 de outubro de 2016.

MARCUZZO, F. F. N.; MELO, D. C. R.; ROCHA, H. M. Distribuição espaço-temporal e sazonalidade das chuvas no Estado de Mato Grosso. Revista Brasileira de Recursos Hídricos, Bento Gonçalves, v. 16, n. 4, p. 154-167, 2011. Acesso por meio de <https://www.abrh.org.br/SGCv3/index.php?PUB=1\&ID=1\&SUMARIO=11>. Acessado em 02 de outubro de 2016. DOI: 10.21168/rbrh.v16n4.p157-167.

MARTINS, J. A.; DALLACORT, R.; INOUE, M. H.; GALVANIN, E. A S; MAGNANI, E. B. Z.; OLIVEIRA, K. C. Caracterização do regime pluviométrico no arco das nascentes do rio Paraguai. Revista Brasileira Meteorologia, São Paulo, v. 26, n. 4, dez. 2011. Acesso por meio de <http://www.scielo.br/pdf/rbmet/v26n4/a13v26n4.pdf>. Acessado em 02 de outubro de 2016. DOI: http://dx.doi.org/10.1590/S0102-77862011000400013.

MORAES, B.C.; COSTA, J.M.N; COSTA, A.C.L.; COSTA, M.H. Variação espacial e temporal da precipitação no Estado do Pará. Acta Amazônica, Manaus, v.35, n.2, p. 207-214, 2005. Acesso por meio de <http://www.scielo.br/pdf/aa/v35n2/v35n2a10.pdf>. Acessado em 02 de outubro de 2016. DOI: http://dx.doi.org/10.1590/S0044-59672005000200010.

MOREIRA, P. S. P.; DALLACORT, R.; MAGALHÃES, R. A.; INOUE, M. H.; STIELER, M. C.; SILVA, D. J.; MARTINS, J. A. Distribuição e probabilidade de ocorrências de chuvas no município de Nova Maringá - MT. Revista de Ciências Agro-Ambientais, Alta Floresta, v. 8, n. 1, p. 9-20, 2010. Acesso por meio de <http://www.unemat.br/revistas/rcaa/docs/vol8/2_artigo_v8.pdf>. Acessado em 02 de outubro de 2016. 
MOURA, Q. L.; RUIVO, M. L. P.; RODRIGUES, H. J. B. Variação sazonal da população de bactérias e fungos e dos teores de nitrato de amônio do solo nos sítios do LPA e PPBIO na Amazônia Oriental. Revista Brasileira de Meteorologia, São Paulo, v. 30, n. 3, p. 265-274, 2015. Acesso por meio de <http://www.scielo.br/pdf/rbmet/v30n3/0102-7786-rbmet-30-03-00265.pdf>.

Acessado em 02 de outubro de 2016. DOI: http://dx.doi.org/10.1590/0102778620140104.

OLIVEIRA, J. P. B.; CECÍLIO, R. A.; XAVIER, A. C.; JASPER, A. P. S.; OLIVEIRA, L. B. Precipitação provável para Alegre-ES através da distribuição de probabilidade Gama. Revista Engenharia Ambiental, Espírito Santo do Pinhal, v. 7, n. 2, p. 204211, 2010. Acesso por meio de $<$ http://ferramentas. unipinhal.edu.br/engenhariaambiental/viewarticle.php?id=461\&lo cale=en $>$. Acessado em 02 de outubro de 2016.

PINTO, L. C.; LIMA, P. L. T.; MELLO, C. R.; SOUZA, Z. R. Probable rainfall in Lambari region, southern Minas Gerais State. Revista Agroambiental, Pouso Alegre, v. 7, n. 4, p. 27-37, 2015. Acesso por meio de <https://agrogeoambiental.ifsuldeminas.edu.br/index.php/Agrogeoambiental/article/vi ew/664/pdf_18>. Acessado em 02 de outubro de 2016. DOI: http://dx.doi.org/10.18406/2316-1817v7n42015664.

PIZZATO, J. A.; DALLACORT, R.; TIEPPO, R. C.; MODOLO, A. J.; CREMON, C.; MOREIRA, P. S. P. Distribuição e probabilidade de ocorrência de precipitação em Cáceres (MT). Revista Pesquisa Agropecuária Tropical, Goiânia, v. 42, n. 2, p. 137-142, 2012. Acesso por meio de <http://www.scielo.br/pdf/pat/v42n2/06.pdf >. Acessado em 02 de outubro de 2016. DOI: http://dx.doi.org/10.1590/S198340632012000200006.

RIBEIRO, B. T.; AVANZI, J. C. Probable monthly raifall for Machado coutry south Minas Gerais State. Revista Agrogeoambiental. Inconfidentes, v. 2, n. 1, p. 117122, 2010. Acesso por meio de $<$ https://agrogeoambiental.ifsuldeminas.edu.br/index.php/Agrogeoambiental/article/vi ew/260 >. Acesso em 02 de outubro de 2016. DOI: http://dx.doi.org/10.18406/2316$1817 \mathrm{v} 2 \mathrm{n} 12010260$.

RIBEIRO, B. T.; AVANZI, J. C.; MELLO, C. R.; LIMA, J. M.; SILVA, L. M. N. Comparação de distribuições de probabilidade e estimativa da precipitação provável para a região de Barbacena, MG. Revista Ciências e Agrotecnologia, Lavras, v. 31, n. 5, p. 1297-1302, 2007. Acesso por meio de <http://www.scielo.br/pdf/cagro/v31n5/04.pdf>. Acessado em 02 de outubro de 2016. DOI: http://dx.doi.org/10.1590/S1413-70542007000500004.

SANTOS, E. H. M.; GRIEBELER, N. P.; OLIVEIRA, L. F. C. Variabilidade espacial e temporal da precipitação pluvial na bacia hidrográfica do Ribeirão João Leite - GO. Engenharia Agrícola, Jaboticabal, v.3, n.1, p.78-89, 2011. Acesso por meio de <http://www.scielo.br/pdf/eagri/v31n1/v31n1a08.pdf>. Acessado em 02 de outubro de 2016. DOI: http://dx.doi.org/10.1590/S0100-69162011000100008.

SANS, L. M. A.; ASSAD, D.; GUIMARÃES, D. P.; AVELAR, G. Zoneamento de riscos climáticos para a cultura do milho na região centro-oeste do Brasil e para o 
estado de Minas Gerais. Revista Brasileira de Agrometeorologia, Passo Fundo, v. 9, n. 3, p. 527-539, 2001. Acesso por meio de <http://www.cnpt.embrapa.br/pesquisa/agromet/pdf/revista/cap17.pdf>. Acessado em 02 de outubro de 2016.

SILVA, J. C.; HELDWEIN, A. B.; FABRINA, B.; TRENTIN, G.; EDENIR, L. Análise de distribuição de chuva para Santa Maria, RS. Revista Brasileira de Engenharia Agrícola e Ambiental, v.11, n.1, p.67-72, 2007. Acesso por meio de <http://www.scielo.br/pdf/rbeaa/v11n1/v11n1a09.pdf>. Acessado em 02 de outubro de 2016. DOI: 10.1590/S1415-43662007000100009.

SOBRINHO, V. F.; RODRIHUES, J. O.; MENDONÇA, L. A. R. Desenvolvimento de equações intencidade-duração-frequência sem dados pluviográficos em regiões semiáridas. Revista Brasileira de Engenharia Agrícola e Ambiental, Campina Grande, v. 18, n. 7, p. 727-734, 2014. Acesso por meio de <http://www.scielo.br/pdf/rbeaa/v18n7/v18n07a09.pdf>. Acessado em 02 de outubro de 2016. DOI: http://dx.doi.org/10.1590/S1415-43662014000700009.

SOCCOL, O. J.; CARDOSO, C. O.; MIQUELLUTI, D. J. Análise da precipitação mensal provável para o município de Lages - SC. Revista Brasileira de Engenharia Agrícola e Ambiental, Campina Grande, v. 14, n. 6, p. 569-574, 2010. Acesso por meio de <http://www.scielo.br/pdf/rbeaa/v14n6/a01v14n6.pdf $>$. Acessado em 02 de outubro de 2016. DOI: http://dx.doi.org/10.1590/S141543662010000600001.

SOUZA, I. F.; LUNDGREN, W. J. C.; NETTO, A. O. Comparação entre distribuições de probabilidades da precipitação mensal no Estado de Pernambuco. Revista Scientia Plena, Aracajú, v. 6, n. 6, p. 1 - 10, 2010. Acesso por meio de $<$ https://www.scientiaplena.org.br/sp/article/view/105/35>. Acessado em 02 de outubro de 2016.

SOUZA, J. L. M.; JERSZURKI D.; DAMAZIO, E. C. Relações funcionais entre precipitação provável e mádia em regiões e climas brasileiros. Revista Pesquisa Agropecuária Brasileira, Brasília, v. 48, n. 7, p. 693-702, 2013. Acesso por meio de $<$ http://www.scielo.br/pdf/pab/v48n7/01.pdf>. Acessado em 02 de outubro de 2016. DOI: http://dx.doi.org/10.1590/S0100-204X2013000700001.

THOM, H. C. S. A note on the gamma distribution. Monthly Weather Review, Whashington, v. 86, p. 117-122, 1958. Acesso por meio de <http://docs.lib.noaa.gov/rescue/mwr/086/mwr-086-04-0117.pdf>. Acessado em 02 de outubro de 2016. DOI: http://dx.doi.org/10.1175/15200493(1958)086<0117:ANOTGD>2.0.CO;2.

ULIANA, E. M.; REIS, E. F.; SILVA, J. G. F; XAVIER, A. C. Precipitação mensal e anual provável para o Estado do Espírito Santo. Revista Irriga, Botucatu, v. 18, n. 1, p. 2013. 139-147, Disponível por meio de <http://biblioteca.incaper.es.gov.br/digital/bitstream/item/392/1/493-2522-1-PB.pdf>. Acessado em 02 de outubro de 2016. DOI: http://dx.doi.org/10.15809/irriga.2013v18n1. 